\title{
Online constraint removal: accelerating MPC with a Lyapunov function
}

\author{
Michael Jost ${ }^{a}$, Gabriele Pannocchia ${ }^{\mathrm{b}}$, Martin Mönnigmann ${ }^{\text {a }}$ \\ ${ }^{a}$ Automatic Control and Systems Theory, Ruhr-Universität Bochum, Bochum, Germany \\ ${ }^{\mathrm{b}}$ Department of Civil and Industrial Engineering, University of Pisa, Pisa, Italy
}

\begin{abstract}
We show how to use a Lyapunov function to accelerate MPC for linear discrete-time systems with linear constraints and quadratic cost. Our method predicts, in the current time step, which constraints will be inactive in the next time step. These constraints can be removed from the online optimization problem of the next time step. The criterion for the detection of inactive constraints is based on the decrease of the Lyapunov function along the trajectory of the controlled system. The criterion is simple, easy to implement in existing MPC algorithms, and its computational cost is small.
\end{abstract}

Key words: Model predictive control, constrained control, linear systems, quadratic programming, Lyapunov methods

\section{Introduction}

Model predictive control (MPC) is an established, practically relevant method for the control of constrained multivariable systems. MPC is computationally expensive, because an optimal control problem must be solved in each time step. For a discrete-time linear system with linear constraints and quadratic cost the optimal control problem is a quadratic program (QP) that is parametrized by the current state of the system. It is known that the solution to this parametric QP is a continuous piecewise affine control law $u(x)$ on a polytopic partition of the state space $[1,2]$. Despite ongoing efforts to improve the algorithms for the calculation [3-6] and fast evaluation [7-9] of these piecewise affine control laws, they can only be calculated and used for small systems with short horizons. Thus, for medium or large systems on-line methods remain the only viable choice.

The discovery of the structure of the solution $u(x)$ prompted research on how to use this structure to accelerate online MPC algorithms. Ferreau and Diehl [10]

\footnotetext{
^ This paper was not presented at any IFAC meeting. Corresponding author M. Mönnigmann, Tel. +49 234 24060; Fax +49234 14155. Funding by Deutsche Forschungsgemeinschaft under grant MO 1086/11-1 is gratefully acknowledged.

Email addresses: michael.s.jost@rub.de (Michael Jost), gabriele.pannocchia@unipi.it (Gabriele Pannocchia), martin.moennigmann@rub.de (Martin Mönnigmann).
}

predict the active set occurring in the next step. Pannocchia et al. $[11,12]$ enumerate the active sets which occurred most frequently during previous operation and store the optimal solution parameters only for those active sets. Jost and Mönnigmann [13,14] calculate state space regions of activity for each constraint offline and use this information online to remove inactive constraints from the QP. In this paper, we also accelerate the online MPC computation by removing constraints from the QP that can be inferred to be inactive before actually solving the QP. In contrast to earlier approaches $[13,14]$ our approach is neither based on the explicit solution nor an approximation thereof. We show that the cost function of the MPC problem can be used to bound the optimal solution for the next time step, if the cost function is a Lyapunov function for the controlled system. The bound on the optimal solution for the next time step only depends on information available at the current time step. While somewhat conservative, this bound can be used to remove some inactive constraints in the next time step, thus simplifying the QP. We stress the proposed method does not just remove constraints from the QP that can never become active, but the set of removed constraints is a function of the current state and thus a function of time.

We introduce the problem class in Section 2. Section 3 establishes the main results described above. An example is discussed in Section 4, and an outlook is given in Section 5 . 


\section{Problem statement and assumptions}

Consider a discrete-time linear time-invariant system

$$
x(t+1)=A x(t)+B u(t),
$$

with state $x(t) \in \mathbb{R}^{n}$, input $u(t) \in \mathbb{R}^{m}$ and matrices $A \in$ $\mathbb{R}^{n \times n}, B \in \mathbb{R}^{n \times m}$, where the pair $(A, B)$ is stabilizable. Assume the system (1) is subject to input and state constraints

$$
u(t) \in \mathbb{U} \subset \mathbb{R}^{m}, \quad x(t) \in \mathbb{X} \subset \mathbb{R}^{n}
$$

for all times $t \in \mathbb{N}$, where $\mathbb{X}$ and $\mathbb{U}$ are compact fulldimensional polytopes that contain the origin in their interiors.

For any initial state $x \in \mathbb{X}$, consider the finite horizon optimal control problem

$$
\begin{aligned}
\mathbb{P}(x): \min _{U, X} & V_{f}(x(N))+\sum_{k=0}^{N-1} \ell(x(k), u(k)) \quad \text { s.t. } \\
x(k+1) & =A x(k)+B u(k), k=0, \ldots, N-1 \\
x(0) & =x, \\
x(k) & \in \mathbb{X}, k=1, \ldots, N-1, \\
x(N) & \in \mathbb{X} f \\
u(k) & \in \mathbb{U}, k=0, \ldots, N-1,
\end{aligned}
$$

where $U=\left(u^{\prime}(0), \ldots, u^{\prime}(N-1)\right)^{\prime}, X=\left(x^{\prime}(1), \ldots, x^{\prime}(N)\right)^{\prime}$, $\mathbb{X}_{f} \subseteq \mathbb{X}$ is a polyhedral terminal set that contains the origin in its interior, and $V_{f}(x)=\frac{1}{2} x^{\prime} P x$, $\ell(x, u)=\frac{1}{2}\left(x^{\prime} Q x+u^{\prime} R u\right)$, where $P \in \mathbb{R}^{n \times n}, P \succ 0$, $Q \in \mathbb{R}^{n \times n}, Q \succeq 0$ and $R \in \mathbb{R}^{m \times m}, R \succ 0$ are the weighting matrices on the terminal state, the states and the inputs, respectively. By eliminating the state variables with (1), the quadratic program (3) can equivalently be written in the form

$$
\min _{U} V(x, U) \text { s. t. } G U-w-E x \leq 0,
$$

where

$$
\begin{aligned}
V(x, U) & =\frac{1}{2}\left(\begin{array}{ll}
x^{\prime} & U^{\prime}
\end{array}\right)\left(\begin{array}{cc}
Y & F \\
F^{\prime} & H
\end{array}\right)\left(\begin{array}{l}
x \\
U
\end{array}\right) \\
& =\frac{1}{2} x^{\prime} Y x+U^{\prime} F^{\prime} x+\frac{1}{2} U^{\prime} H U,
\end{aligned}
$$

$H \in \mathbb{R}^{m N \times m N}, Y \in \mathbb{R}^{n \times n}, F \in \mathbb{R}^{n \times m N}, G \in \mathbb{R}^{q \times m N}$, $E \in \mathbb{R}^{q \times n}, w \in \mathbb{R}^{q}$, and where $q$ denotes the number of inequality constraints in (3) and (4). It can be shown that $Y^{\prime}=Y, H^{\prime}=H$ and $H \succ 0$, if $R \succ 0$, $P \succ 0$ and $Q \succeq 0$. Consequently, (4) is a strictly convex quadratic program. This implies the solution to (4), and equivalently to (3), is unique if it exists. We note for later use that $(6)$ can be rewritten as $V(x, U)=\frac{1}{2}(U+$ $\left.H^{-1} F^{\prime} x\right)^{\prime} H\left(U+H^{-1} F^{\prime} x\right)+\frac{1}{2} x^{\prime} Y x-\frac{1}{2} x^{\prime} F H^{-1} F^{\prime} x$ by completing the squares. For brevity we write this expression for $V(x, U)$ as

$$
V(x, U)=\frac{1}{2}\left\|U+H^{-1} F^{\prime} x\right\|_{H}^{2}+\frac{1}{2}\|x\|_{Y-F H^{-1} F^{\prime}}^{2},
$$

where $\|\xi\|_{M}^{2}=\xi^{\prime} M \xi$ and $\|\xi\|^{2}=\xi^{\prime} \xi$ for any vector $\xi \in \mathbb{R}^{s}$ and symmetric matrix $M \in \mathbb{R}^{s \times s}$. We show that $Y-F H^{-1} F^{\prime} \succ 0$ in the appendix.

Problem (3) may not have a solution for all $x \in \mathbb{X}$. Let $\mathcal{X} \subseteq \mathbb{X}$ be the set of initial conditions $x$ such that (3) has a solution. For any $x \in \mathcal{X}$, let $U^{\star}(x)$ and $u^{\star}(x)$ refer to the optimal solution to (3) and its first element, respectively. Denote the corresponding optimal value of (4) by $V^{\star}(x)$ and recall this is equal to the optimal value of (3). Using (6) we may express $V^{\star}(x)$ as

$$
V^{\star}(x)=\frac{1}{2} x^{\prime} Y x+U^{\star \prime}(x) F^{\prime} x+\frac{1}{2} U^{\star \prime}(x) H U^{\star}(x) .
$$

Under the assumptions stated so far, $\mathcal{X}$ is convex, the functions $U^{\star}: \mathcal{X} \rightarrow \mathbb{U}^{N}$ and $u^{\star}: \mathcal{X} \rightarrow \mathbb{U}$ are continuous and piecewise affine, and $V^{\star}: \mathcal{X} \rightarrow \mathbb{R}$ is continuous, convex and piecewise quadratic [2]. Let the symbol $x^{+}$ denote the predicted successor state for the controlled system, i.e.,

$$
x^{+}=A x+B u^{\star}(x) .
$$

We make the following assumption throughout the paper.

Assumption 1 The optimal value function $V^{\star}(x)$ of $\mathbb{P}(x)$ is a Lyapunov function for the closed-loop system (8), i.e., there exist strictly positive constants $a_{1}, a_{2}$ and $a_{3}$ such that $x \in \mathcal{X}$ implies

$$
\begin{gathered}
a_{1}\|x\|_{2}^{2} \leq V^{\star}(x) \leq a_{2}\|x\|_{2}^{2} \\
V^{\star}\left(x^{+}\right)-V^{\star}(x) \leq-a_{3}\|x\|_{2}^{2} .
\end{gathered}
$$

This assumption is guaranteed to hold if the terminal constraint set $\mathbb{X}_{f}$ and cost functions $\ell(\cdot), V_{f}(\cdot)$ satisfy control invariance conditions [15]. Note that nominal exponential stability of the origin of the closed-loop system follows from Assumption 1.

\subsection{Notation and preliminaries}

Let $\mathcal{Q}=\{1, \ldots, q\}$ denote the index set of the constraints of (4). For any matrix $M \in \mathbb{R}^{q \times t}$, let $M^{i}$ and $M^{\mathcal{W}}$ be the row vector and submatrix of row vectors indicated by $i \in \mathcal{Q}$ and the ordered subset $\mathcal{W} \subseteq \mathcal{Q}$, respectively. The $i$-th constraint in (4) is called inactive at the optimum, 
if $G^{i} U^{\star}(x)<w^{i}+E^{i} x$, and active if $G^{i} U^{\star}(x)=w^{i}+E^{i} x$, where $U^{\star}(x)$ is the optimal solution to (4) at state $x$. We say a constraint $i \in \mathcal{Q}$ is a priori known to be inactive for a particular $x \in \mathcal{X}$ if we know $G^{i} U^{\star}(x)<w^{i}+E^{i} x$ before having solved (4) for $U^{\star}(x)$.

Recall that a quadratic form with symmetric positive definite matrix $M$ defines an ellipsoid $\left\{\xi \in \mathbb{R}^{s} \mid \xi^{\prime} M \xi \leq\right.$ $1\} \subset \mathbb{R}^{s}$ centered at the origin and, for any $\xi_{0} \in \mathbb{R}^{s}$, an ellipsoid

$$
\left\{\xi \in \mathbb{R}^{s} \mid\left(\xi-\xi_{0}\right)^{\prime} M\left(\xi-\xi_{0}\right) \leq 1\right\}
$$

centered at $\xi_{0}$. Let $\lambda_{\min }(M)$ and $\lambda_{\max }(M)$ denote the smallest and largest eigenvalue of $M$, respectively. We state an important property of ellipsoids in the following lemma.

Lemma 2 Let $M \in \mathbb{R}^{s \times s}$ be a symmetric positive definite matrix, and consider any $\xi \in \mathbb{R}^{s}$ and $\alpha \geq 0$. Then

$$
\xi^{\prime} M \xi \leq \alpha^{2} \quad \text { implies }\|\xi\| \leq \frac{\alpha}{\sqrt{\lambda_{\min }(M)}} .
$$

PROOF. For any symmetric $M \in \mathbb{R}^{s \times s}$

$$
\lambda_{\min }(M) \xi^{\prime} \xi \leq \xi^{\prime} M \xi
$$

for all $\xi \in \mathbb{R}^{s}\left[16\right.$, Lemma 8.4.3]. Therefore $\xi^{\prime} M \xi \leq \alpha^{2}$ implies $\lambda_{\min }(M) \xi^{\prime} \xi \leq \alpha^{2}$. Since $M$ is positive definite by assumption, $\lambda_{\min }(M)>0$ and the claim follows.

\section{Reduced equivalent MPC problem}

Assume the optimal control problem (4) has been solved for the current initial condition $x$, and hence the optimal sequence of controls $U^{\star}(x)$ has been determined. The predicted successor state $x^{+}$of the controlled system is given by (8). In the next time step we need to solve (4) for $x^{+}$to find $U^{\star}\left(x^{+}\right)$. The present section explains how to simplify (4) by removing constraints that can be shown to be inactive for $x^{+}$before actually solving (4) for $x^{+}$.

\subsection{Constraint removal}

We first show how a bound $\left\|U^{\star}(x)\right\|<c$ can be used to test whether one or some of the constraints in (4) are inactive.

Lemma 3 Let $x \in \mathcal{X}$ be arbitrary and assume there exists $c \geq 0$ such that $\left\|U^{\star}(x)\right\| \leq c$. If, for any $i \in \mathcal{Q}$,

$$
\left\|G^{i}\right\| c<E^{i} x+w^{i}
$$

then constraint $i$ is inactive at the optimal solution to (3) for $x$, i.e., $G^{i} U^{\star}(x)<E^{i} x+w^{i}$.
PROOF. Consider the relations

$$
G^{i} U^{\star}(x) \leq\left|G^{i} U^{\star}(x)\right| \leq\left\|G^{i}\right\|\left\|U^{\star}(x)\right\| \leq\left\|G^{i}\right\| c,
$$

which hold, because $\xi \leq|\xi|$ for all $\xi \in \mathbb{R}$, according to Cauchy and Schwarz's inequality, and because $\left\|U^{\star}(x)\right\| \leq c$ by assumption, respectively. Together with (12) this implies $G^{i} U^{\star}(x)<E^{i} x+w^{i}$, which proves the claim.

Constraints that are a priori known to be inactive at $U^{\star}(x)$ for the current $x \in \mathcal{X}$ may be removed from the parametric quadratic program. Note that we do not need to know all inactive constraints at $U^{\star}(x)$ in Lemma 4 below but only a subset $\mathcal{J}(x)$ thereof. In other words, if $\mathcal{I}(x) \subseteq \mathcal{Q}$ is the set of inactive constraints at $U^{\star}(x)$, then Lemma 4 applies to any $\mathcal{J}(x) \subseteq \mathcal{I}(x)$.

Lemma 4 Let $x \in \mathcal{X}$ be arbitrary. Assume there exist $c>0$ and a set $\mathcal{J}(x) \subseteq \mathcal{Q}$ such that (12) holds for every $i \in \mathcal{J}(x)$. Then the reduced quadratic program

$$
\begin{aligned}
& \min _{U} V(x, U) \text { s.t. } \\
& \qquad G^{i} U-w^{i}-E^{i} x \leq 0, \quad i \in \mathcal{Q} \backslash \mathcal{J}(x)
\end{aligned}
$$

has the same unique solution $U^{\star}(x)$ as (4).

Lemma 4 can be proven by showing that the KKT conditions of (4) and (13) have the same unique solution, taking into account that Lagrange multipliers of inactive constraints are zero.

\subsection{Bound on $\left\|U^{\star}\left(x^{+}\right)\right\|$from $U^{\star}(x)$}

For any $x \in \mathcal{X}$, the optimal solution $U^{\star}(x)$ can be exploited to obtain a bound on the norm of the optimal solution $U^{\star}\left(x^{+}\right)$in the next time step. This is stated concisely in Proposition 5 and Corollary 6.

Proposition 5 Let $x \in \mathcal{X}$ be arbitrary, assume $U^{\star}(x)$ has been found, and let $x^{+}=A x+B u^{\star}(x)$. The optimal control sequence $U^{\star}\left(x^{+}\right)$for the predicted closed-loop successor state $x^{+}$lies in the ellipsoid

$$
\mathcal{U}^{+}(x)=\left\{U \in \mathbb{R}^{m N} \mid\left\|U+H^{-1} F^{\prime} x^{+}\right\|_{\frac{1}{2} H}^{2} \leq \rho(x)\right\}
$$

where

$$
\rho(x)=V^{\star}(x)-\frac{1}{2}\left\|x^{+}\right\|_{Y-F H^{-1} F^{\prime}}^{2}
$$

is strictly positive for all $x \in \mathcal{X} \backslash\{0\}$ and $\rho(0)=0$.

PROOF. We first show that $\rho(x)>0$ for all $x \in \mathcal{X} \backslash\{0\}$ by contradiction. Assume there exists an $x \in \mathcal{X} \backslash\{0\}$ 
such that $\rho(x) \leq 0$. By definition of $\rho(x)$ this implies $V^{\star}(x) \leq \frac{1}{2}\left\|x^{+}\right\|_{Y-F H^{-1} F^{\prime}}^{2}$. Relation (10) yields $V^{\star}\left(x^{+}\right)<V^{\star}(x)$ for any $x \in \mathcal{X} \backslash\{0\}$, therefore $V^{\star}\left(x^{+}\right)<\frac{1}{2}\left\|x^{+}\right\|_{Y-F H^{-1} F^{\prime}}^{2}$, or equivalently

$$
V^{\star}\left(x^{+}\right)-\frac{1}{2}\left\|x^{+}\right\|_{Y-F H^{-1} F^{\prime}}^{2}<0 .
$$

Substituting

$$
\begin{aligned}
V^{\star}\left(x^{+}\right)=\frac{1}{2} \| U^{\star}\left(x^{+}\right)+H^{-1} & F^{\prime} x^{+} \|_{H}^{2} \\
& +\frac{1}{2}\left\|x^{+}\right\|_{Y-F H^{-1} F^{\prime}}^{2}
\end{aligned}
$$

which results from combining (7) and $V^{\star}\left(x^{+}\right)=$ $V\left(x^{+}, U^{\star}\left(x^{+}\right)\right)$, yields

$$
\frac{1}{2}\left\|U^{\star}\left(x^{+}\right)+H^{-1} F^{\prime} x^{+}\right\|_{H}^{2}<0,
$$

which contradicts $H \succ 0$ and thus proves that $\rho(x)$ is strictly positive on $\mathcal{X} \backslash\{0\}$. To prove that $\rho(0)=0$, we recall that $U^{\star}(0)=0$, and hence $x^{+}=0$.

It remains to show that $U^{\star}\left(x^{+}\right) \in \mathcal{U}^{+}(x)$. Consider the relation $V^{\star}\left(x^{+}\right) \leq V^{\star}(x)$ again, which follows from (10). Substituting (16) yields

$$
\left\|U^{\star}\left(x^{+}\right)+H^{-1} F^{\prime} x^{+}\right\|_{\frac{1}{2} H}^{2}+\frac{1}{2}\left\|x^{+}\right\|_{Y-F H^{-1} F^{\prime}}^{2} \leq V^{\star}(x),
$$

which is equivalent to the inequality in (14) and therefore proves $U^{\star}\left(x^{+}\right) \in \mathcal{U}^{+}(x)$.

A bound on $\left\|U^{\star}\left(x^{+}\right)\right\|$can now be stated based on Proposition 5.

Corollary 6 Let $x \in \mathcal{X}$ be arbitrary, assume $U^{\star}(x)$ has been found, let $x^{+}=A x+B u^{\star}(x)$, and let $\rho(x)$ be as in (15). Then the norm of $U^{\star}\left(x^{+}\right)$, the input sequence that is optimal in the next time step, is bounded above by

$$
\begin{aligned}
& \bar{U}(x)=\sqrt{\frac{2 \rho(x)}{\lambda_{\min }(H)}}+\left\|H^{-1} F^{\prime}\left(A x+B u^{\star}(x)\right)\right\|, \\
& \text { i.e., }\left\|U^{\star}\left(x^{+}\right)\right\| \leq \bar{U}(x) .
\end{aligned}
$$

PROOF. Let $U \in \mathcal{U}^{+}(x)$ be arbitrary. According to Lemma 2 this implies

$$
\left\|U+H^{-1} F^{\prime} x^{+}\right\| \leq \sqrt{\frac{2 \rho(x)}{\lambda_{\min }(H)}},
$$

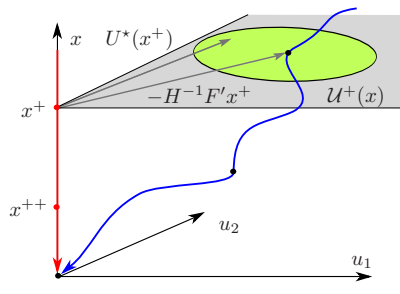

(a)

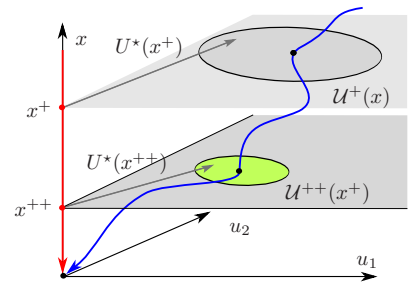

(b)
Fig. 1. Interpretation of Corollary 6 for a hypothetical system with two inputs and one state: (a) Acc. to Proposition 5 the optimal input sequence for the next time step, $U^{\star}\left(x^{+}\right)$, lies in the ellipsoid $\mathcal{U}^{+}(x)$ that is determined by the current state and the current optimal input sequence. Corollary 6 essentially states that an upper bound $\bar{U}(x)$ on $\left\|U^{\star}\left(x^{+}\right)\right\|$is given by the sum of the distance $\left\|H^{-1} F^{\prime} x^{+}\right\|$of the ellipsoids center to the origin and the length of the longest semi-major axis of the ellipsoid (second and first term in (17), respectively). (b) Since the controlled system is asymptotically stable, we have $x \rightarrow 0$ for $k \rightarrow \infty$. The ellipsoid $\mathcal{U}^{+}(x)$ shrinks along any trajectory of the controlled system $(\rho(x) \rightarrow 0)$, and its center tends to the origin $\left(H^{-1} F^{\prime} x^{+} \rightarrow 0\right)$. Consequently, the upper bound $\bar{U}(x)$ on $\left\|U\left(x^{+}\right)\right\|$converges to zero. The symbol $x^{++}$denotes the successor state to $x^{+}$, i.e., $x^{++}=A x^{+}+B u^{\star}\left(x^{+}\right)$.

where we used $\lambda_{\min }(k M)=k \lambda_{\min }(M)$, which holds for any $k>0$ and $M \succ 0$. As a further preparation note that

$$
\|a\|=\|a+b-b\| \leq\|a+b\|+\|-b\|=\|a+b\|+\|b\|,
$$

for any two elements $a, b$ of a normed vector space, where the second relation in (19) is the triangle inequality. Relation (19) implies $\|a\|-\|b\| \leq\|a+b\|$. By applying this relation to $U$ and $H^{-1} F^{\prime} x^{+}$we find $\|U\|-$ $\left\|H^{-1} F^{\prime} x^{+}\right\| \leq\left\|U+H^{-1} F^{\prime} x^{+}\right\|$. Together with (18) this implies $\|U\|-\left\|H^{-1} F^{\prime} x^{+}\right\| \leq \sqrt{2 \rho(x) / \lambda_{\min }(H)}$, which proves the claim.

Figure 1 gives a graphical interpretation of the ellipsoid $\mathcal{U}^{+}(x)$ and the bound $\left\|U^{\star}(x)\right\| \leq \bar{U}(x)$ defined in (14) and (17), respectively. Note that the shape of $\mathcal{U}^{+}(x)$ is defined by the matrix $H$, while its center and size are a function of the current state $x$ and its optimal control sequence $U^{\star}(x)$. Recall that the closed-loop successor state $x^{+}$is uniquely defined by $x$ and $U^{\star}(x)$ according to $(8)$.

\subsection{A simple state independent bound}

Since $\mathbb{U}$ is compact by assumption there exists a bounded hyperrectangle that contains it, i.e., there exist $\underline{u}_{i}<\bar{u}_{i}$, $i=1, \ldots, m$ such that

$$
\mathbb{U} \subseteq\left[\underline{u}_{1}, \bar{u}_{1}\right] \times \cdots \times\left[\underline{u}_{m}, \bar{u}_{m}\right] .
$$


This yields the simple bound on $\left\|U^{\star}(x)\right\|$ stated in the following lemma. While obvious, the resulting bound is important, because it is, in contrast to $\bar{U}(x)$ introduced in (17), independent of the state $x$.

Lemma 7 Consider the optimal control problem (3) and assume $\underline{u}_{i}, \bar{u}_{i}, i=1, \ldots, m$ are as in $(20)$. Let $\hat{u}=$ $\left(\max \left(\left|\underline{u}_{1}\right|,\left|\bar{u}_{1}\right|\right), \ldots, \max \left(\left|\underline{u}_{m}\right|,\left|\bar{u}_{m}\right|\right)\right)^{\prime}$. Then $\left\|U^{\star}(x)\right\|$ is bounded above by

$$
\hat{U}=\sqrt{N}\|\hat{u}\|
$$

i.e., $\left\|U^{\star}(x)\right\| \leq \hat{U}$ holds for all $x \in \mathcal{X}$.

PROOF. From $U=\left(u(0)^{\prime}, \ldots, u(N-1)^{\prime}\right)^{\prime}$ we infer $\|U\|^{2}=\sum_{k=0}^{N-1}\|u(k)\|^{2}$. Since $u(k) \in \mathbb{U} \subseteq\left[\underline{u}_{1}, \bar{u}_{1}\right] \times \cdots \times$ $\left[\underline{u}_{m}, \bar{u}_{m}\right]$ implies $\|u(k)\|^{2} \leq \sum_{l=1}^{m}\left(\max \left(\left|\underline{u}_{l}\right|,\left|\bar{u}_{l}\right|\right)\right)^{2}$ for all $k$, we have $\|U\|^{2} \leq N \sum_{l=1}^{m}\left(\max \left(\left|\underline{u}_{l}\right|,\left|\bar{u}_{l}\right|\right)\right)^{2}$.

Since the bound defined in (21) does not depend on $x$, it can be calculated offline.

\subsection{Reduced MPC problem}

It is easy to prove the following proposition based on the previous results.

Proposition 8 Let $x, x^{+}, U^{\star}(x), \bar{U}(x), \rho(x)$ and $\hat{U}$ be as in Corollary 6 and Lemma 7 , respectively. Let

$$
\mathcal{J}=\left\{i \in \mathcal{Q} \mid\left\|G^{i}\right\| \min (\hat{U}, \bar{U}(x))<E^{i} x^{+}+w^{i}\right\} .
$$

Then the optimal sequence $U^{\star}\left(x^{+}\right)$for the predicted closed-loop successor state $x^{+}$can be calculated from the reduced $Q P$

$$
\min _{U} V\left(x^{+}, U\right) \text { s.t. } G^{i} U-w^{i}-E^{i} x^{+} \leq 0, i \in \mathcal{Q} \backslash \mathcal{J},
$$

because the constraints $G^{i} U^{\star}\left(x^{+}\right) \leq E^{i} x^{+}+w^{i}$ are inactive for all $i \in \mathcal{J}$.

PROOF. Corollary 6 and Lemma 7 imply that $\left\|U^{\star}\left(x^{+}\right)\right\| \leq \bar{U}(x)$ and $\left\|U^{\star}\left(x^{+}\right)\right\| \leq \hat{U}$, respectively. Therefore, $\left\|U^{\star}\left(x^{+}\right)\right\| \leq \min (\bar{U}(x), \hat{U})$. Now let $i \in \mathcal{J}$ be arbitrary. From applying Lemma 3 to $x^{+}$for $c=\min (\bar{U}(x), \hat{U})$ we infer constraint $i$ is inactive at $U^{\star}\left(x^{+}\right)$, which proves the last statement in the proposition. By applying Lemma 4 the first claim results.

Proposition 8 obviously results in the original full QP (4) if $\mathcal{J}=\emptyset$, i.e., if no constraints can be inferred to be inactive with Corollary 6 and Lemma 7.

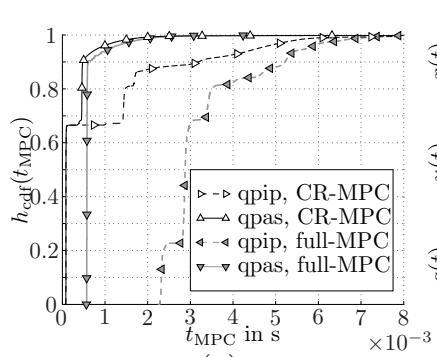

(a)

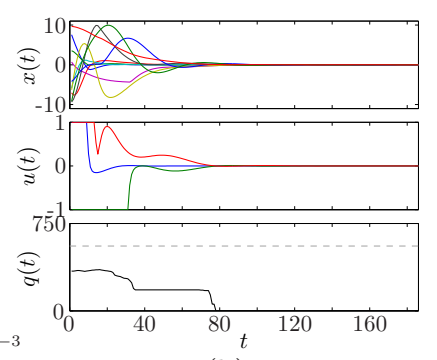

(b)
Fig. 2. (a) Cumulative distribution functions of the times required to solve (4) with an interior-point solver (qpip, dashed) and an active-set solver (qpas, solid), both with and without constraint removal (black and gray, respectively). (b) States $x(t)$, inputs $u(t)$ and number of constraints $q(t)$ of the system for $0 \leq t \leq 186$. The gray dashed line represents the number of constraints of the full QP.

\section{Example}

We apply MPC with and without constraint removal to a system of the form (1) with $n=10$ states and $m=3$ inputs. The system is a minimal representation of the transfer function matrix

$$
G(s)=\left[\begin{array}{ccc}
\frac{-5 s+1}{36 s^{2}+6 s+1} & \frac{0.5 s}{8 s+1} & 0 \\
0 & \frac{0.1(-10 s+1)}{s(8 s+1)} & \frac{-0.1}{\left(64 s^{2}+6 s+1\right) s} \\
\frac{-2 s+1}{12 s^{2}+3 s+1} & 0 & \frac{2(-5 s+1)}{16 s^{2}+2 s+1}
\end{array}\right]
$$

discretized with zero-order hold and sampling time $T_{s}=$ $1 \mathrm{~s}$ (see [17] for the state space model). The example is nontrivial in that there exist zeroes in the right half plane and two uncontrollable states must be removed to obtain the $n=10$ minimal representation. Reparametrizing the inputs as proposed in [18] results in a well-conditioned matrix $H$ with $\lambda_{\max }(H)=1.70$ and $\lambda_{\min }(H)=0.68$. We impose the constraints $-10 \leq x_{i} \leq 10, i=1, \ldots, 10$ and $-1 \leq u_{j} \leq 1, j=1, \ldots, 3$ and set $Q=I^{n \times n}$, $R=0.25 I^{m \times m}$ and $N=30$. The terminal weighting matrix $P$ is the solution of the discrete-time algebraic Riccati equation. After removing redundant constraints a quadratic program of the form (4) results with 90 variables and 556 constraints (see e.g. [19, Sec. 4.1.1, p. 128] on redundant constraints).

We randomly choose feasible initial points $x \in \mathcal{X}$ and calculate closed-loop trajectories until $\|x(t)\|_{2} \leq 10^{-3}$. A total of 771,678 QPs was solved in each of the four cases summarized in Fig. 2. Four cases arise because $\mathrm{MPC}$ with constraint removal (CR-MPC for short) and MPC without constraint removal (full-MPC for short) are both combined with an active-set and an interiorpoint solver. ${ }^{1}$ The results of our numerical experiments

\footnotetext{
1 We used the primal-dual interior-point solver qpip and the dual active-set solver qpas from the QPC solver library [20].
} 


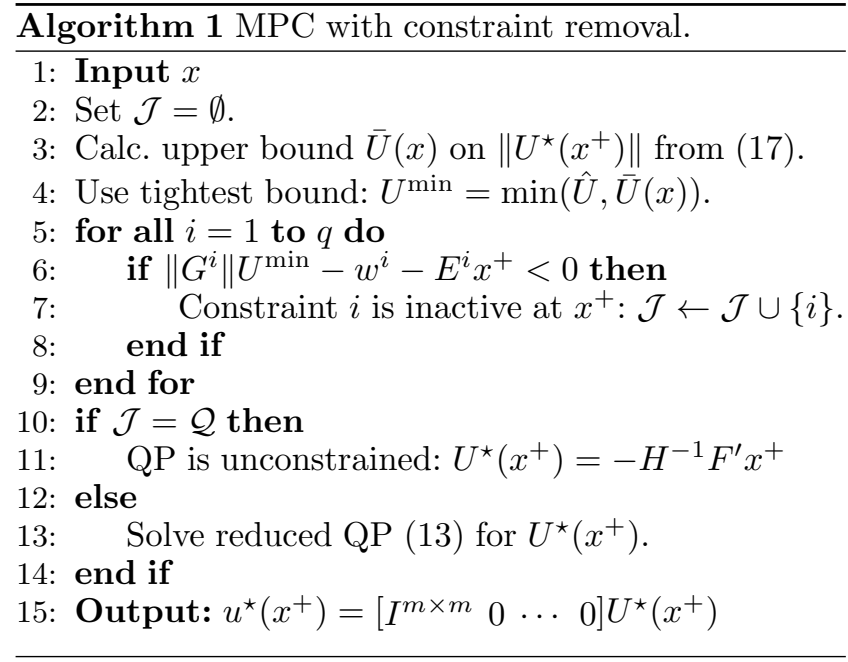

are summarized in Fig. 2(a) in terms of $h_{\mathrm{cdf}}\left(t_{\mathrm{MPC}}\right)$, the cumulative distribution function (cdf) of the time $t_{\mathrm{MPC}}$ required to solve (4). The cdf $h_{\mathrm{cdf}}\left(t_{\mathrm{MPC}}\right)$ is defined as the fraction of QPs (4) that are solved in time $t_{\mathrm{MPC}}$ or less.

About $65 \%$ of the QPs are found to be unconstrained by CR-MPC. No optimization problem needs to be solved at all in these cases, but the optimal input results from the state feedback law of the unconstrained case (cf. line 11 in Alg. 1). CR-MPC provides the control law very quickly in these cases, which results in the leftmost shoulders of the black curves in Fig. 2(a). Note that the computation times do not depend on the solver in these cases. Consequently, the leftmost shoulders of the black curves coincide.

For both the active-set solver and the interior-point solver CR-MPC outperforms full-MPC in the sense that the cdf for CR-MPC lies to the left and above the corresponding one for full-MPC. For about $88 \%$ (92\%) of all QPs CR-MPC calculates the control law faster than the shortest time among all QPs required by full-MPC with the interior-point solver (active-set solver). ${ }^{2}$ The average computation time is reduced by about $71 \%$ $(57 \%)$ for the interior-point solver (active-set solver). More details are given in [17].

Figure 2(b) shows the states $x(t)$, inputs $u(t)$ and the number of constraints $q(t)$ for one of the random initial values. It is easy to see that the number of constraints $q(t)$ quickly decreases along the trajectory, until only the unconstrained quadratic program remains. For this particular trajectory, the bound $\hat{U}$ is used for $t \leq 22$, while $\bar{U}(x)$ is used for $t>22$. Note that $\rho(x)$ and thus $\bar{U}(x)$ cannot be calculated in the first time step, since the value of the objective function at the previous time

\footnotetext{
2 These figures can be found in Fig. 2(a) by drawing a line parallel to the ordinate that cuts the abscissa at the smallest computation time achieved by full-MPC (gray curve).
}

step is not known. Consequently, during the first time step only the bound $\hat{U}$ can be used.

We carried out similar numerical experiments for 36 example-solver combinations (six dynamical systems and MPC problems with $q=258$ to $q=1950$ combined with six QP solvers). We summarize some important aspects: (i) In all but the smallest example MPC with constraint removal outperformed the same implementation without constraint removal in the same sense as in the example shown here. When the smallest example in [17] is combined with a dual active-set solver, MPC with constraint removal is sometimes faster but sometimes slower than without constraint removal. This results, because the overhead for detecting inactive constraints and setting up the reduced problem (13) is not compensated by the savings from solving the reduced QP if the number of constraints is small. (ii) While larger reductions result for interior-point solvers than for active-set solvers in general, the computational times for the active-set solvers are reduced in all but the case summarized in (i). ${ }^{3}$ (iii) The numerical experiments clearly indicate that redundant constraints need not be removed a priori, but constraint removal detects and removes them efficiently. We present results in Fig. 2 for a case in which we did remove redundant constraints a priori to show that constraint removal detects inactive constraints beyond the redundant ones.

\subsection{Robustness of the proposed approach}

We briefly investigated the robustness of the proposed approach. Specifically, we added a uniformly distributed disturbance $-0.5 \leq \Delta x_{i} \leq 0.5, i=1, \ldots, 10$ to the state, determined the reduced QP with the nominal state $\bar{U}(x)$ but solved it for the corresponding disturbed state, and compared the resulting control law to the case without constraint removal. Despite the disturbance, the optimal input resulted in $99.7 \%$ of the cases. Essentially, this is due to the fact that the bound $\bar{U}(x)$ is conservative and therefore inherently robust.

\section{Conclusion and outlook}

We presented a simple but effective way to accelerate model predictive control for linear systems with linear constraints and quadratic cost. Existing MPC implementations can easily be enhanced with the proposed method, since it does not depend on details of the optimization algorithm, and since only very simple additional calculations are necessary. Simulation studies show that the presented method is able to reduce the average calculation times significantly.

\footnotetext{
${ }^{3}$ One may conjecture that no savings could be obtained for dual active-set solvers. Results shown here and in [17] show that this is not true, however. See [17, Sect. 5.3] for an explanation.
} 
Future research must investigate the robustness of the proposed approach and address the extension to nonlinear MPC. Moreover, it has been shown for small examples [13] that MPC can considerably be accelerated by anticipating active constraints in addition to inactive constraints. Finally, we so far exploit the decrease of the Lyapunov function for only one time step. Further accelerations may be possible for longer time spans.

\section{Appendix}

Proof of $Y-F H^{-1} F^{\prime} \succ 0$

According to Assumption 1, there exists an $a_{1}>0$ such that $V^{\star}(x) \geq a_{1}\|x\|_{2}^{2}$ for all $x \in \mathcal{X}$. Together with $V^{\star}(x)=V\left(x, U^{\star}(x)\right)$ this implies $V\left(x, U^{\star}(x)\right)>0$ for all $x \in \mathcal{X} \backslash\{0\}$. Because $V\left(x, U^{\star}(x)\right) \leq V(x, U)$ by definition of $U^{\star}(x)$, we also have $V(x, U)>0$ for all $x \in \mathcal{X} \backslash\{0\}$ and all feasible $U$, or equivalently for all elements of $\mathcal{P}=\left\{(U, x) \in \mathbb{R}^{N m} \times \mathbb{R}^{n} \mid G U-E x \leq w\right\}$ with $x \neq 0$. We therefore showed that the quadratic form (5) is positive definite on a full-dimensional set. This implies the matrix that defines this quadratic form, which we denote by $\Gamma$, is positive definite. Applying the Schur complement (see e.g. [21, Thm. 7.7.6, p. 472]) to $\Gamma$ yields $Y \succ F H^{-1} F^{\prime}$, or equivalently $Y-F H^{-1} F^{\prime} \succ 0$.

\section{References}

[1] M. Seron, G. Goodwin, and J. DeDona, "Characterisation of receding horizon control for constrained systems," Asian Journal of Control, vol. 5, pp. 271 - 286, 2003.

[2] A. Bemporad, M. Morari, V. Dua, and E. N. Pistikopoulos, "The explicit linear quadratic regulator for constrained systems," Automatica, vol. 38, pp. 3-20, 2002.

[3] A. Gupta, S. Bhartiya, and P. Nataraj, "A novel approach to multiparametric quadratic programming," Automatica, vol. 47, no. 9, pp. 2112 - 2117, 2011.

[4] P. Patrinos and H. Sarimveis, "A new algorithm for solving convex parametric quadratic programs based on graphical derivatives of solution mappings," Automatica, vol. 46, no. 9, pp. 1405 - 1418, 2010.

[5] S. Columbano, K. Fukuda, and C. Jones, "An outputsensitive algorithm for multi-parametric LCPs with sufficient matrices," AMS CRM Proceedings and Lecture Notes, vol. 48, pp. 73-102, 2009.

[6] M. Mönnigmann and M. Jost, "Vertex based calculation of explicit MPC laws." in Proceedings of the 2012 American Control Conference, Montreal, Canada, 2012, pp. 423-428.

[7] M. Mönnigmann and M. Kastsian, "Fast explicit model predictive control with multiway trees," in Proceedings of IFAC World Congress 2011, Milano, Italy, 2011, pp. 13561361.

[8] F. Bayat, T. A. Johansen, and A. A. Jalali, "Using hash tables to manage the time-storage complexity in a point location problem: Application to explicit model predictive control," Automatica, vol. 47, no. 3, pp. 571 - 577, 2011.

[9] P. Tøndel, T. A. Johansen, and A. Bemporad, "Evaluation of piecewise affine control via binary search tree," Automatica, vol. 39, pp. $945-950,2003$.
[10] H. Ferreau, H. Bock, and M. Diehl, "An online active set strategy to overcome the limitations of explicit MPC," International Journal of Robust and Nonlinear Control, vol. 18, pp. 816-830, 2008.

[11] G. Pannocchia, J. B. Rawlings, and S. J. Wright, "Fast, large-scale model predictive control by partial enumeration," Automatica, vol. 43, pp. 852-860, 2007.

[12] G. Pannocchia, S. J. Wright, and J. B. Rawlings, "Partial enumeration MPC: Robust stability results and application to an unstable CSTR," Journal of Process Control, vol. 21, no. 10, pp. 1459-1466, 2011.

[13] M. Jost and M. Mönnigmann, "Accelerating online MPC with partial explicit information and linear storage complexity in the number of constraints," in Proceedings of the European Control Conference 2013, Zurich, Switzerland, 2013, pp. 3540.

$[14] \_$, "Accelerating model predictive control by online constraint removal," in Proceedings of the Conference on Decision and Control 2013, Florence, Italy, 2013, pp. 57645769.

[15] D. Q. Mayne, J. B. Rawlings, C. V. Rao, and P. O. M. Scokaert, "Constrained model predictive control: Stability and optimality," Automatica, vol. 36, pp. 789 - 814, 2000.

[16] D. S. Bernstein, Matrix Mathematics - Theory, Facts and Formulas. Princeton University Press, USA, 2009.

[17] M. Jost, G. Pannocchia, and M. Mönnigmann, "Simulation Studies to Online constraint removal: accelerating MPC with a Lyapunov function," Ruhr-Universität Bochum, Tech. Rep., 2014.

[18] J. A. Rossiter, G. Kouvaritakis, and M. J. Rice, "A nummerically robust state-space approach to stablepredictive control stratigies," Automatica, vol. 34, pp. 65-73, 1998.

[19] S. Boyd and L. Vandenberghe, Convex Optimization, S. Edition, Ed. New York, NY, USA: Cambridge University Press, 2009.

[20] D. A. Wills, QPC - Quadratic Programming in $C$, School of Electrical Engineering and Computer Science, University of Newcastle, 2009. [Online]. Available: http://sigpromu.org/quadprog/index.html

[21] R. A. Horn and C. R. Johnson, Matrix Analysis, 23rd ed. Cambridge University Press, 2010. 